\title{
Medical image segmentation using Fuzzy C-Mean (FCM) and user specified data
}

\begin{abstract}
Image segmentation is one of the most important parts of clinical diagnostic tools. Medical images mostly contain noise and inhomogeneity. Therefore, accurate segmentation of medical images is a very difficult task. However, the process of accurate segmentation of these images is very important and crucial for a correct diagnosis by clinical tools. We proposed a new clustering method based on Fuzzy C-Mean (FCM) and user specified data. In the postulated method, the color image is converted to grey level image and anisotropic filter is applied to decrease noise; User selects training data for each target class, afterwards, the image is clustered using ordinary FCM. Due to inhomogeneity and unknown noise some clusters contain training data for more than one target class. These clusters are partitioned again. This process continues until there are no such clusters. Then, the clusters contain training data for a target class assigned to that target class; mean of intensity in each class is considered as feature for that class, afterwards, feature distance of each unsigned cluster from different class is found then unsigned clusters are signed to target class with least distance from. Experimental result is demonstrated to show effectiveness of new method.
\end{abstract}

Keyword: Image segmentation; Supervised method; MRI; FCM; Re-clustering 